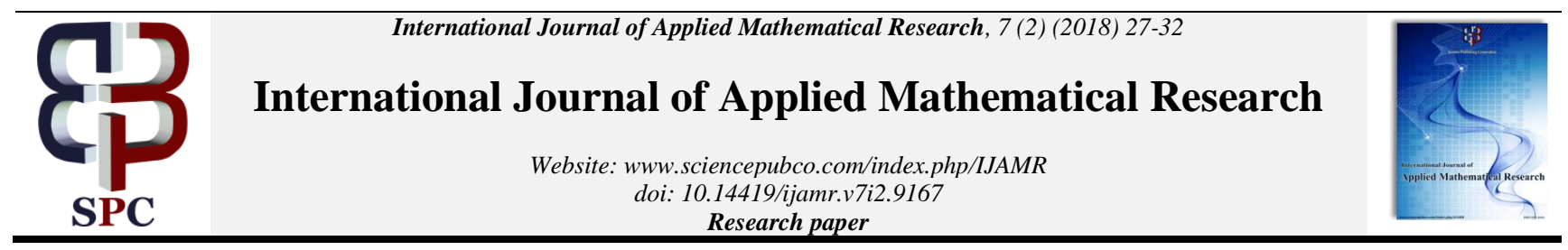

\title{
Finite time stability of linear fractional order dynamical system with variable delays
}

\author{
Jackreece P. C ${ }^{1}$, Aniaku S. E ${ }^{2}$ \\ ${ }^{1}$ Department of Mathematics and Statistics, University of Port Harcourt, Port Harcourt, Nigeria \\ ${ }^{2}$ Department of Mathematics and Statistics, University of Nigeria, Nsukka, Nigeria \\ *Corresponding author E-mail: prebo.jackreece@uniport.edu.ng
}

\begin{abstract}
In this paper, some sufficient condition ensuring finite time stability are derived for a class of linear fractional order dynamical system with variable delay using generalized Gronwall inequality as well as classical Bellman-Gronwall inequality.
\end{abstract}

Keywords: Dynamical System; Fractional Calculus; Finite Time Stability; Time Varying Delays.

\section{Introduction}

Time delay systems are important class of dynamical systems. Time delays are very often encountered in different technical systems such as electric, pneumatic and hydraulic networks, chemical processes, long transmission lines, control systems etc. [19], [20]. These delays are due to transportation of materials, energy or information, [54], [14]. The existence of time delays may cause undesirable system transient response and frequently the source of instability. Consequently, the question of stability of these class of systems is of theoretical and practical importance, see [2], [37], [56].

Numerous remarkable results relating to the stability of time delay systems have been published with particular emphasis on the application of Frequency domain techniques [25], Lyapunov methods, or idea of matrix measure [33], [41], small-gain-base methods [17].

In practice one is not only interested in system stability (in the sense of Lyapunov), but also in bounds of system trajectories. A system could be stable but still completely useless if it possesses undesirable transient performance. Thus, it may be useful to consider the stability of such systems with respect to certain subset of the state space which are defined a priori in a given problem. Besides that, it is of particular significance to consider the behavior of dynamical system only over a finite time interval. For this purpose, the concept of finite time stability and practical stability has been used. A system is said to be finite time stable if, given a bound on the initial condition, its state does not exceed a certain threshold within a specified time interval. To verify the finite time stability of systems, several authors have developed different techniques to investigate stability criteria, see [1], [7], [8], [23], [24], [26], [36], [37], [42], [49] and references therein.

Fractional calculus was first introduced 300 years ago and dates back to the works of Leibnitz, Liouville, Riemann, Grunwald, and Letnikov [28], [48]. Fractional calculus is a generalization of the traditional (integer order) calculus in which the order of the derivatives and integers can be any real or complex number. In recent years, the study of fractional order derivative has attracted increasing interest due to an important role it plays not only in mathemat- ics, but also in physics, engineering, control systems, dynamical systems and in particular in the modeling of many natural phenomena [24], [50], [28], [53]. There are two aspects that essentially differentiates fractional order models and integer order, which makes it more realistic to characterize real world physical systems by fractional order state equation. First for the integer order derivative indicates a variation of a certain attributes at a particular time for a physical or mechanical process, while fractional order is concerned with the whole-time domain. Second, integer order derivatives describe the local properties of a certain position for physical process, while fractional order derivative is related to the whole space, [3], ]18].

However, in many real world physical systems, fractional calculus is more feasible than integer calculus to model the behaviors of such system. For example, fractional electrical networks [39], fractional order Schrodinger equation [43], fractional oscillator equation [46], fractional Lotka-Volterra equation [18], robotics [15]. In particular, stability analysis is one of the most fundamental and important issues for systems.

It is well known that the analysis of the stability of fractional systems is more complex than that of classical differential equation, since fractional derivatives are nonlocal and have more weakly singular kernels. Algebraic criteria for stability analysis of classical (integer order) system, such as Hurwitz and Routh criteria or Jury's, cannot be used for fractional order system, because fractional order systems do not have characteristics polynomials but pseudo polynomial with rational power multivalued function. Thus, there remain only geometric methods (Nyquist) which can be used for the stability check for bounded input bounded output. Different techniques have been proposed in the investigation of the stability for various fractional dynamical system, such as analytical approach [4], [27], fixed point theorem [9], [10], [51], the Lyapunov method [34], [35], linear matrix inequality [47], Gronwall inequality [12], [30].

Recently there have been advances in control theory of fractional order dynamical systems for different kinds of stability. [38] considered the structural stability result of fractional differential equations with applications to control processing from both algebraic and analytical point of views. In their paper [11], they analyzed the stability of linear fractional differential system with multiple

Copyright $\odot 2018$ Jackreece P. C, Aniaku S. E. This is an open access article distributed under the Creative Commons Attribution License, which permits unrestricted use, distribution, and reproduction in any medium, provided the original work is properly cited. 
time delays. Based on the characteristic equation defined by using Laplace transform, several stability criterions are derived.

In [31], they extend some basic results of finite and practical stability of linear, continuous fractional order invariant time delay systems with delay in the state by proposing a stability test procedure using Bellman-Gronwall theorem. [12] obtained sufficient condition for the finite time stability of a class of fractional singular time delay systems by giving the Mittag-Leffler estimate of the solution for an equivalent system. Also, finite time stability analysis of fractional order time delay systems is studied in [30, 32] Our aim in this paper is to develop sufficient condition for the finite time stability of a class of fractional order systems with time varying delays.

\section{Preliminaries}

Consider the equation

$$
\begin{aligned}
& \dot{x}(t)=A_{0}(t) x(t)+A_{1}(t) x(t-\tau(t))+B(t) u(t) \\
& x(t)=\psi_{x}(t), \quad \forall t \in\left[-\tau_{M}, 0\right]
\end{aligned}
$$

Where $x(t) \in R^{n}$ is the state vector, $u(t) \in R^{m}$ is the input control vector, $A_{0}(t), A_{1}(t) \in R^{n \times n}, B(t) \in R^{m}$ are matrices of bounded variation, $\tau(t)$ is time varying delays, $\psi_{x} \in C\left([-\tau, 0], R^{n}\right)$ is an admissible initial state and $C\left(\left[-\tau_{k}, 0\right], R^{n}\right)$ is a Banach space of continuous function mapping the interval $\left[-\tau_{M}, 0\right]$ into $R^{n}$ which converges uniformly, and the norm defined by

$$
\|\varphi\|=\sup |\varphi(\theta)|
$$

The system behavior is defined over the time interval $I=[0, T]$ where $\mathrm{T}$ is a positive number.

For the time invariant sets $S_{()}$, used as bounds of the system trajectories are assumed to be bounded, open and connected. Let $S_{\delta}$ be a given set of all allowable states of the system for $\forall t \in I$. Let $S_{\varepsilon}$ be the set of all initial states of the system such that $S_{\delta} \subseteq S_{\varepsilon}$ and $S_{\gamma}$ denote the set of all allowable control actions. The sets $S_{\delta}$ and $S_{\varepsilon}$ are connected and a priori known. Generally, the set

$$
S_{\rho}=\left\{x(t):\|x(t)\|_{Q}^{2}<\rho\right\}, \quad \rho \in[\delta, \varepsilon]
$$

The time varying delay is assumed to satisfy the following condition

$$
0 \leq \tau(t) \leq \tau_{M}
$$

Where $\tau_{M}$ represents the maximum delays. Before proceeding further, we will introduce the following definitions and theorems which will be used in the next section.

Matrix measures have been extensively studied in $[13,21]$ and it is used to estimate upper bounds of matrix exponential. The following theorem relates an upper bound of a matrix exponential to its matrix measures.

Theorem 2.1: For any matrix $A \in R^{n \times n}$ the estimate

$$
\|\exp (A(t))\| \leq \exp (\mu(A)(t))
$$

Holds [12], [22].

Theorem 2.2: The matrix norm or Lozinskii logarithm norm of a $n \times n$ matrix $A$ is
$\mu(A)=\lim _{h \rightarrow 0} \frac{|I+h A|-I}{h}$

Where $\|()$.$\| is any matrix norm compatible with some vector norm$ $|x|_{\text {. }}$. The matrix measure defined in theorem 2.2 has three variants depending on the norm utilized in the definition.

It is assumed that the usual smoothness condition is satisfied by system (2.1) so that there will be no difficulty with the question of existence, uniqueness and continuity of solutions with respect to initial data.

Before stating our results, we introduce the concept of finite-time stability for time-delay system (2.1). This concept can be formalized through the following definition.

Definition 2.1: Time delayed control system is finite time stable with respect to $\left\{S_{\varepsilon}, S_{\delta}, T,\|()\|,. \mu\left(A_{0}\right) \neq 0\right\}, \quad \delta<\varepsilon$ if and only if; $\psi_{x}(t) \in S_{\delta}, \forall t \in[-\tau, 0]$

And

$u(t) \in S_{\gamma}, \forall t \in T$

Implies

$x\left(t: t_{0}, x_{0}\right) \in S_{\varepsilon}, \forall t \in[0, T]$

See [6], [31].

Definition 2.2: For any real matrix $A=\left(a_{i j}\right)_{n \times n}$, its matrix measure is defined as

$\mu_{\rho}(A)=\lim _{\varepsilon \rightarrow 0^{+}} \frac{\|I-\varepsilon A\|-1}{\varepsilon}$

Where $\|.\|_{\rho}$ denotes the matrix norm in $R^{n \times n}, I$ is the identity matrix and $\rho=[1,2, \infty]$ norm. the matrix norms are defined as follows

$\left.\|A\|_{1}=\max _{j}\left\{\sum_{i=1}^{n} \mid a_{i j}\right\}\right\},\|A\|_{2}=\sqrt{\lambda_{\max }\left(A^{T} A\right)}$

And

$\|A\|_{\infty}=\max _{i}\left\{\sum_{j=1}^{n}\left|a_{i j}\right|\right\}$

Lemma 2.1: For the definition of matrix measure, for any $A, B \in R^{n \times n}, \rho=1,2, \infty$, we have

1) $-\|A\|_{\rho} \leq \mu_{\rho}(A) \leq\|A\|_{\rho}$

2) $\mu_{\rho}(\alpha A)=\alpha \mu_{\rho}(A), \quad \forall \alpha>0$

3) $\mu_{\rho}(A+B) \leq \mu_{\rho}(A)+\mu_{\rho}(B)$

Definition 2.3: System given by eq. (2.1) satisfying the initial condition $x(t)=\psi_{x}(t),-\tau_{M} \leq t \leq 0$ is finite time stable w.r.t. $\left\{\delta, \varepsilon, \gamma, t_{0}, J\right\}, \delta<\varepsilon$ if and only if $\left\|\psi_{x}\right\|_{c}<\delta$ and $\|u(t)\|<\gamma_{u}, \forall t \in J$ imply $\|x(t)\|<\varepsilon, \forall t \in J$. 


\section{Fundamentals of fractional calculus}

At first, the differential and integral operators can be generalized into one fundamental $D_{t}^{\alpha}$ operator $\mathrm{t}$ which is known as fractional calculus [5], [28], [44], [45].

${ }_{a} D_{t}^{\alpha}= \begin{cases}\frac{d^{\alpha}}{d t^{\alpha}} & \Re(\alpha)>0 \\ 1 & \Re(\alpha)=0 \\ \int_{a}^{t}(d \tau)^{-\alpha} & \Re(\alpha)<0\end{cases}$

There are many ways to define fractional derivatives and integrals. The definition generally used in recent studies are, GrunwaldLetinkov, Riemann-Liouville and Caputo definitions.

Definition 3.1: The Grunwald-Letnikov (GL) fractional derivative of order $\alpha, \alpha>0$ and fractional integral of order $\alpha, \alpha>0$ of a continuous function $f(t)$ defined on the interval $[a, b]$ are defined by

$$
\begin{aligned}
& { }_{a}^{G L} D_{t}^{\alpha} f(t)=\lim _{h \rightarrow 0} \frac{1}{h^{\alpha}} \sum_{k=0}^{[(t-\alpha) h h]}(-1)^{k}\left(\begin{array}{l}
\alpha \\
k
\end{array}\right) f(t-k h), \\
& \left(\begin{array}{l}
\alpha \\
k
\end{array}\right)=\frac{\Gamma(k-\alpha)}{\Gamma(-\alpha) \Gamma(k+1)}
\end{aligned}
$$

And the fractional integral as

$$
\begin{aligned}
{ }_{a}^{G L} D_{t}^{\alpha} f(t)=\sum_{k=0}^{n-1} & \frac{f^{(k)}(0) t^{-\alpha+k}}{\Gamma(-\alpha+k+1)} \\
& +\frac{1}{\Gamma(n-\alpha)} \int_{a}^{t} \frac{f^{(n)}(s)}{(t-s)^{\alpha-n+1}} d s
\end{aligned}
$$

Where $a$ and $t$ are limits of the operator, [(.)] denotes the integer part of (.) and $\Gamma($.$) is the Euler's gamma function that generalizes$ factorial for non-integer arguments:

$\Gamma(z)=\int_{0}^{\infty} e^{-t} t^{z-1} d t, \Gamma(z+1)=\Gamma(z), z=x+i y$

One basic property of the gamma function is that it satisfies the functional equation

$$
\Gamma(z)=z \Gamma(z), \Rightarrow \Gamma(n+1)=n(n+1) !=n !
$$

Definition 3.2: Let $[a, b]$ be a finite interval, $-\infty<a<b<\infty$, $[a, b] \subset R$ and $f(t)$ be a continuous function defined on $[a, b]$, the Riemann-Liouville fractional derivative of order $\alpha$ is given by

$$
\begin{aligned}
& { }_{a} D_{t}^{\alpha} f(t) \\
& =\frac{1}{\Gamma(n-\alpha)} \frac{d^{n}}{d t^{n}} \int_{a}^{t} \frac{f(s)}{(t-s)^{\alpha-n+1}} d s
\end{aligned}
$$

For $(n-1<a<n)$ and $\Gamma($.$) is Euler's gamma function.$

Closely related to Riemann-Liouville Fractional order derivative is the fractional integral defined by

${ }_{a} D_{t}^{-\alpha} f(t)$

$$
=\frac{1}{\Gamma(\alpha)} \int_{a}^{t} \frac{f(s)}{(t-s)^{1-\alpha}} d s, \quad \alpha<0
$$

In [3] it has been proposed that the integer order (classical) derivative of function $\mathrm{x}$, as are commonly used in initial value problem with integer order equations be incorporated.
Definition 3.3 [5]: The Caputo fractional derivative of order $\alpha<0$ of a function $f:(0, \infty) \rightarrow R$ can be written as

$$
\begin{aligned}
&{ }_{0}^{c} D_{t}^{\alpha}[f(t)]= \frac{d^{\alpha} f}{d t^{\alpha}} \\
&=\frac{1}{\Gamma(n-\alpha)} \int_{0}^{t} \frac{f^{(n)}(s)}{(t-s)^{n-\alpha-1}} d s \\
& n-1<\alpha<n, \quad f^{(n)}(s)=\frac{d^{n} f}{d s^{n}}
\end{aligned}
$$

Some properties of Riemann-Liouville and Caputo derivatives are recalled here, [40], [55].

Property 3.1

When $0<\alpha<1$, we have

$$
{ }_{t_{0}}^{c} D_{t}^{\alpha} x(t)={ }_{t_{0}} D_{t}^{\alpha} x(t)-\frac{x\left(t_{0}\right)}{\Gamma(1-\alpha)}\left(t-t_{0}\right)^{-\alpha}
$$

In particular, if ${ }_{t_{0}}^{c} D_{t}^{\alpha} x(t)={ }_{t_{0}} D_{t}^{\alpha} x(t)$

Property 3.2

For $v>1$, we have

$$
{ }_{t_{0}} D_{t}^{\alpha}\left(t-t_{0}\right)^{v}=\frac{\Gamma(1+v)}{(1+v-\alpha)}\left(t-t_{0}\right)^{v-\alpha}
$$

In particular, if $0<\alpha<1$ and $x(t)=\left(t-t_{0}\right)^{v}$ then, from property 3.1, we have

${ }_{{ }_{0}}^{c} D_{t}^{\alpha}\left(t-t_{0}\right)^{v}=\frac{\Gamma(1+v)}{\Gamma(1+v-\alpha)}\left(t-t_{0}\right)^{v-\alpha}$

Property 3.3

From the definition of Caputo derivation eq. (3.5) when $0<\alpha \leq 1$ we have

$I_{t_{0} t_{0}}^{\alpha c} D_{t}^{\alpha} x(t)=x(t)-x\left(t_{0}\right)$

Where

$\left(I_{t_{0}}^{\alpha} f\right)(t)=\frac{1}{\Gamma(\alpha)} \int_{t_{t}}^{t} \frac{f(s)}{(t-s)^{1-\alpha}} d s, t>t_{0}$

Property 3.4

Fractional order differentiation is a linear operator:

$D^{\alpha}(\lambda f(t)+\mu g(t))=\lambda D^{\alpha} f(t)+\mu D^{\alpha} g(t)$

Also, the chain rule has the form

$$
\frac{d^{\beta} f(g(t))}{d t^{\beta}}=\sum_{k=0}^{\infty}\left(\begin{array}{l}
\beta \\
k
\end{array}\right)_{\Gamma}\left(\frac{d^{\beta-k}}{d t^{\beta-k}} I\right) \frac{d^{k}}{d t^{k}} f(g(t))
$$

Where $k \in N$ and $\left(\begin{array}{l}\beta \\ k\end{array}\right)_{\Gamma}$ are the coefficients of the generalized binomial

$\left(\begin{array}{l}\beta \\ k\end{array}\right)_{\Gamma}=\frac{\Gamma(1+\beta)}{\Gamma(1+k) \Gamma(1-k+\beta)}$

There are also two functions that play an important role in the study of stability of FDE's

Definition 3.4: The Mittag-Leffler function is defined by 


$$
E_{\alpha}(z)=\sum_{k=0}^{\infty} \frac{z^{k}}{\Gamma(k \alpha+1)}
$$

Where $\operatorname{Re}(z)>0, z \in C$. the two parameter Mittag-Leffler function is defined by

$$
E_{\alpha, \beta}(z)=\sum_{k=0}^{\infty} \frac{z^{k}}{\Gamma(k \alpha+\beta)}
$$

Where $\operatorname{Re}(z)>0$, and $\beta \in C, z \in C$

Definition 3.5: The $\alpha$-exponential function is defined by

$$
e_{\alpha}^{\lambda z}=z^{\alpha-1} E_{\alpha, \alpha}\left(\lambda z^{\alpha}\right)
$$

Where $z \in C \backslash 0, \operatorname{Re}(\alpha)>0$ and $\lambda \in C . E_{\alpha, \alpha}($.$) is the two parameter$ Mittag-Leffler function. Mittage-Leffler function is frequently used in the solution of fractional order system and is a generalization of the exponential function.

\section{Main result}

Here, a class of linear dynamical system with time varying delays in the state of the form

$$
\begin{aligned}
& D_{t}^{\alpha}=\frac{d^{\alpha}}{d t^{\alpha}} \\
& =A_{0} x(t)+A_{1} x(t-\tau(t))+B u(t)
\end{aligned}
$$

With initial condition $x(t)=\psi_{x}(t)$, where the time varying delays satisfy eq. (2.2) and $D_{t}^{\alpha}$ denotes Caputo fractional derivative of order $\alpha, 0<\alpha<1$ is considered.

\section{Lemma 4.1: (Bellman-Gronwall inequality [16], [52])}

Suppose $x(t)$ and $a(t)$ are nonnegative and locally integrable on $0 \leq t<t, T \leq \infty$, and $g(t)$ is nonnegative continuous function defined on $0 \leq t<t, g(t) \leq M, M$ a constant, $\alpha>0$ with

$x(t)=a(t)+g(t) \int_{0}^{t}(t-s)^{\alpha-1} x(s) d s$

On this interval, then:

$x(t) \leq a(t)$

$+\int_{0}^{t}\left(\frac{(g(t) \Gamma(\alpha))}{\Gamma(n \alpha)}(t-s)^{n \alpha-1}\right) d s, \quad 0 \leq t<T$

Theorem 4.1: The dynamical system eq. (4.1) satisfying the initial condition $x(t)=\psi_{x}(t),-\tau_{M} \leq t \leq 0$ is finite time stable w.r.t. $\left\{\delta, \varepsilon, \alpha_{u}, J\right\}, \delta<\varepsilon$ if the following condition is satisfied

$$
\begin{aligned}
& \left(1+\frac{\mu_{\Sigma}\left(t-t_{0}\right)^{\alpha}}{\Gamma(\alpha+1)}\right) e^{\frac{\sigma_{\Sigma}\left(t-t_{0}\right)^{\alpha}}{\Gamma(\alpha+1)}} \\
& +\frac{\gamma^{*}\left[b\left(t-t_{0}\right)+\tau_{M}^{*}\right]}{\Gamma(\alpha+1)} \leq \varepsilon / \delta
\end{aligned}
$$

Where $\gamma^{\cdot}=\alpha_{u} \alpha_{0} / \delta, \Gamma($.$) Euler's gamma function.$

Proof

In accordance with the property of fractional order $0<\alpha<1$, the solution can be obtained in the form of an equivalent Volterra integral equation:

$$
\begin{aligned}
& x(t)=x\left(t_{0}\right) \\
& +\frac{1}{\Gamma(\alpha)} \int_{t_{0}}^{t}(t-s)^{\alpha-1}\left(\begin{array}{l}
A_{0} x(s) \\
+A_{1} x(s-\tau(s)) \\
+B u(s)
\end{array}\right) d s
\end{aligned}
$$

To obtain an estimate of the solution we apply the norm $\|()$.$\| to eq.$ (4.2) and using appropriate property of the norm, the following applies:

$$
\begin{aligned}
& \|x(t)\| \leq\left\|x\left(t_{0}\right)\right\| \\
& +\frac{1}{\Gamma(\alpha)} \int_{t_{0}}^{t}(t-s)^{\alpha-1}\left\|\begin{array}{l}
A_{0} x(s) \\
+A_{1} x(s-\tau(s)) \\
+B u(s)
\end{array}\right\| d s
\end{aligned}
$$

Also, applying the norm $\|()$.$\| to eq. (4.1), it holds:$

$\left\|\frac{d^{\alpha} x(t)}{d t^{\alpha}}\right\| \leq\left\|A_{0}\right\|\|x(t)\|+\left\|A_{1}\right\|\|x(t-\tau(t))\|+\|B\|\|u(t)\|$

$$
\begin{aligned}
& \leq\left(\sigma_{\max }\left(A_{0}\right)\right)\|x(t)\| \\
& +\left(\sigma_{\max }\left(A_{1}\right)\right)\|x(t-\tau(t))\|+\|B\|\|u(t)\|
\end{aligned}
$$

Where $\|A\|$ denotes the induced norm of matrix A, considering

$$
\begin{aligned}
& \|x(t-\tau(t))\| \\
& \leq \sup \left\{\left\|x\left(t^{*}\right)\right\|: t^{*} \in\left[t-\tau_{\max }, t\right]\right\}
\end{aligned}
$$

Applying the inequality eq. (4.5), eq. (4.4) can be written as

$$
\begin{aligned}
& \left\|\frac{d^{\alpha} x(t)}{d t^{\alpha}}\right\| \leq\left(\sigma_{\max }\left(A_{0}\right)\right)\|x(t)\| \\
& +\left(\sigma_{\max }\left(A_{1}\right)\right) \sup _{t^{*} \in\left[t-\tau_{M}, t\right]}\left\|x\left(t^{*}\right)\right\|+\|b\|\|u(t)\| \\
& \leq \sigma_{\sum \max } \sup _{i^{*} \in\left[t-\tau_{u}, t\right]}\left\|x\left(t^{*}\right)\right\|+b\|u(t)\|
\end{aligned}
$$

So,

$$
\begin{aligned}
\left\|\frac{d^{\alpha} x(t)}{d t^{\alpha}}\right\| & \\
\leq & \sigma_{\Sigma}\left(\sup _{t^{*} \in\left[t-\tau_{M}\right]}\left\|x\left(t^{*}\right)\right\|+\left\|\psi_{x}\right\|_{c}\right) \\
& +b\|u(t)\|
\end{aligned}
$$

Where

$$
\sigma_{\Sigma}=\sigma_{M a x}\left(A_{0}\right)+\sigma_{M a x}\left(A_{1}\right), b=\sigma_{M a x}(B)
$$

Combining eq. (4.7) with eq. (4.3) yields

$$
\begin{aligned}
& \|x(t)\| \leq\left\|x\left(t_{0}\right)\right\|
\end{aligned}
$$

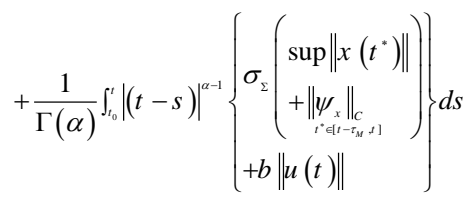




$$
\begin{aligned}
& \leq\left\|\psi_{x}\right\|_{C} \\
& +\frac{1}{\Gamma(\alpha)} \int_{t_{0}}^{t} \mid(t-s)^{\alpha-1}\left\{\begin{array}{l}
\sigma_{\Sigma}\left(\begin{array}{l}
\sup _{t^{*} \in\left[t_{t}, t\right]}\left\|x\left(t^{*}\right)\right\| \\
+\left\|\psi_{x}\right\|_{c}
\end{array}\right) \\
+b\|u(t)\|
\end{array}\right\} d s \\
& \leq\left\|\psi_{x}\right\|_{C}\left(1+\frac{\sigma_{\Sigma}\left(t-t_{0}\right)^{\alpha}}{\Gamma(\alpha+1)}\right)+\frac{\alpha_{u \alpha_{0}}}{\Gamma(\alpha+1)}\left(b\left(t-t_{0}\right)+\tau_{M}^{*}\right) \\
& +\frac{\sigma_{\Sigma}}{\Gamma(\alpha)} \int_{t_{0}}^{t}\left|(t-s)^{\alpha-1}\right| \sup _{t^{*} \in\left[t \tau_{M}, t\right]}\left\|x\left(t^{*}\right)\right\| d s
\end{aligned}
$$

Let

$$
\begin{aligned}
a(t)= & \left\|\psi_{x}\right\|_{c}\left(1+\frac{\sigma_{\Sigma}\left(t-t_{0}\right)^{\alpha}}{\Gamma(\alpha+1)}\right) \\
& +\frac{\alpha_{u} \alpha_{0}}{\Gamma(\alpha+1)}\left[b\left(t-t_{0}\right)+\tau_{M}^{*}\right] \\
g(t) & =\frac{\sigma_{\Sigma}}{\Gamma(\alpha)}
\end{aligned}
$$

By eq. (4.8), we have

$$
\begin{aligned}
& \|x(t)\| \leq a(t) \\
& +g(t) \int_{t_{0}}^{t}\left|(t-s)^{\alpha-1}\right| \sup _{t^{*} \in\left[t \tau_{M}, t\right]}\left\|x\left(t^{*}\right)\right\| d s
\end{aligned}
$$

Obviously, the right-hand side of eq. (4.10) is a nondecreasing continuous function defined on $[0, \mathrm{~T}]$, hence

$$
\begin{aligned}
& \sup _{t^{*} \in\left[t-\tau_{M}, t\right]}\left\|x\left(t^{*}\right)\right\| \leq a(t) \\
& +g(t) \int_{t_{0}}^{t}\left|(t-s)^{\alpha-1}\right| \sup _{t^{*} \in\left[t-\tau_{M}, t\right]}\left\|x\left(t^{*}\right)\right\| d s
\end{aligned}
$$

Applying the generalized Gronwall inequality, lemma 4.1, leads to

$$
\begin{aligned}
& \|x(t)\| \leq \sup _{t^{*} \in\left[t-\tau_{M}, t\right]}\left\|x\left(t^{*}\right)\right\| \leq a(t) e^{g(t) \int_{t_{0}}^{f_{0}}\left[\left(t-t_{0}\right)^{\alpha-1} \mid d s\right.} \\
& =a(t) e^{\frac{\sigma_{\sin }\left(t-t_{0}\right)^{\alpha}}{\Gamma(\alpha+1)}}
\end{aligned}
$$

And the relation

$$
\begin{aligned}
\|x(t)\| \leq \delta & \left(1+\frac{\sigma_{\Sigma}\left(t-t_{0}\right)^{\alpha}}{\Gamma(\alpha+1)}\right) e^{\frac{\sigma_{\Sigma}\left(t-t_{0}\right)^{\alpha}}{\Gamma(\alpha+1)}} \\
& +\frac{\alpha_{u} \alpha_{0}}{\Gamma(\alpha+1)}\left[b\left(t-t_{0}\right)+\tau_{M}^{*}\right]
\end{aligned}
$$

Hence by the basic conditions of theorem (4.1), eq. (4.1) yields

$$
\|x(t)\|<\varepsilon \forall t \in J
$$

This completes the proof.

\section{Conclusion}

In this article, stability results of a class of linear fractional order systems with time varying delays in the state was considered. Stability criteria for this class of system was derived by applying Bellman-Gronwall theorem where sufficient conditions of finite time stability are obtained.

\section{References}

[1] F. Amato, M. Ariola, C. Cosentino, C. T. Abdallah, and P. Dorato, Necessary and sufficient conditions for finite-time stability of linear systems. Proc. American Control Conference, Denver, Colorado, USA (2003), 4452-4456.

[2] E. K. Boukas and N. F. Al-Muthairi, Delay-dependent stabilization of singular linear systems with delays. International Journal of Innovative Computing, Information and Control, 2(2), (2006), 283291.

[3] M. Caputo, Linear models of dissipation whose Q is almost frequency independent, II. Geophys. J. R. Astron. Soc. 13, (1967), 529-539. https://doi.org/10.1111/j.1365-246X.1967.tb02303.x.

[4] Y. Q. Chen and K. L. Moore, Analytical Stability bound for a class of delayed fractional order dynamic system, Nonlin. Dyn. Vol. 29, (202), 191-200.

[5] S. Das, Functional fractional calculus, Academic press, San Diego (1999).

[6] D. L. Debeljkovi, M. P. Lazarevic, D. Koruga, S. A. Milinkovic, M. B. Jovanovic, and L. A. Jacic, Further Results on Non-Lyapunov stability of the linear nonautonomous systems with delayed state, Facta Universitatis, Mechanics, Automatic Control and Robotic series, 3(11), (2001), 231-241.

[7] D. L. Debeljkovi, S. B. Stojannovic, G. V. Simeunovic and N. J. Dimitrijevic, Further results on stability of singular time delay systems in the sense of non-Lyapunov: A new delay dependent conditions, Automatic Control and Information Sciences, 2(1), (2014) 13-19.

[8] D. L. Debeljkovic, S. B. Stojanovic, and A. M. Jovanovic, Further results on finite time and practical stability of linear continuous time delay systems. FME Transactions, 41(3), (2013), 241-249.

[9] W. Deng, Smoothness and stability of the solution for nonlinear fractional differential equation, Nonlinear Anal. 72(3-4), (2010), 1768- 1777. https://doi.org/10.1016/j.na.2009.09.018.

[10] W. Deng, and C. Li, Numerical schemes for fractional ordinary differential equations. Numerical Modelling, Dr. Peep Miidla (Ed), ISBN: 978-953-51-0219-9, InTech, Retrieved from http://cdn.intechopen.com/pdfs-wm/33091.pdf, (2012).

[11] W. Deng, C. Li, and J. Lü, Stability analysis of linear fractional differential system with multiple time delays. Nonlinear Dynamics, 48(4), (2007), 409-416. https://doi.org/10.1007/s11071-006-9094-0.

[12] P. Denghao, and J. Wei, Finite time stability analysis of fractional singular time delay systems, Advances in difference equation, 2014/1/259.

[13] C. A. Desoer, and M. Vidyasagar, (1975). Feedback Systems: Input- Output properties. Academic Press, New York, (1975).

[14] L. Dugard, and E. I. Verriest, Stability and control of time delay systems Springer-Verlag, (1997).

[15] N. M. F. Ferreira, F. B. Duarte, F. Miguel, M. G. Marcos, and J. A. T. Machado, Application of fractional calculus in the dynamical analysis and control of mechanical manipulators. Fractional calculus and applied analysis, 11(1), (2008), 91-113.

[16] J. K. Hale, Functional differential equations, Springer, New York, (1971). https://doi.org/10.1007/978-1-4615-9968-5.

[17] K. Gu, V. I. Kharitonov, and J. Chen, Stability of time delay systems, Boston, Birkhauser, (2003). https://doi.org/10.1007/978-14612-0039-0.

[18] R. Hilfer, Application of fractional calculus in Physics, World Scientific Singapore, (2000) https://doi.org/10.1142/3779.

[19] Y. Hong, Y. Xu, and J. Huang, Finite time control for robot manipulation, Systems and Control Letters, 46, (2002), 243-253. https://doi.org/10.1016/S0167-6911(02)00130-5.

[20] Y. Hong, Finite time Stabilization and Stabilizability of a class of controllable systems. Systems and Control Letters, 46, (2002), 231236. https://doi.org/10.1016/S0167-6911(02)00119-6.

[21] G. D. Hu, and M. Z. Liu, The weighted logarithm matrix norm and bounds of the matrix exponential. Linear Algebra Appl., 390, (2004), 145-154. https://doi.org/10.1016/j.laa.2004.04.015.

[22] G. D. Hu and T. Mitsui, Bounds of the matrix eigenvalues and its exponential by lyapunov equation. Kybernetika, 48(5), (2012), $865-878$.

[23] P. C. Jackreece, Finite time stability of linear control system with multiple delays, Control Theory and Informatics, Vol. 6(3), (2016), 69-72.

[24] P. C. Jackreece, Finite time stability of linear control system with constant delay in the state, International Journal of Mathematics and Statistics Invention(IJMI), Vol.5(2), (2017), 49-52.

[25] H. Jia, X. Cao, X. Yu, and P. Zhang, A simple approach to determine power system delay margin, in Proceedings of the IEEE PES 
general meeting, Montreal, Quebec, (2007), 1-7 https://doi.org/10.1109/PES.2007.385467.

[26] N. A. Kablar, and D. L. Debeljkovic, Non-Lyapunov stability of linear singular systems: Matrix measure approach. Preprints 5th IFAC Symposium on Low Cost Automation, Shenyang, China, September 8- 10, TS13, (1998), 16-20.

[27] E. Kaslik, and S. Sivasundaram, An analytical and numerical methods for the stability analysis of linear fractional delay differential equations Journal of computational and Applied Mathematics, 236(16), (2012),

4027-4041. https://doi.org/10.1016/j.cam.2012.03.010.

[28] A. A. Kilbas, H. M. Srivastava, and J. J. Trujillo, Theory and applications of fractional differential equations, Elsevier, New York, (2006).

[29] V. V. Kulish, and J. L. Lage, Application of fractional calculus to Mechanics, Journal of Fluids Engineering, Vol. 124, No. 3, (2002), 803-806. https://doi.org/10.1115/1.1478062.

[30] M. P. Lazarevic, Finite time stability analysis of $P D^{\alpha}$ fractional control of robotic time delay systems, Mech. Res. Commun.33 (2), (2006), 269-

279. https://doi.org/10.1016/j.mechrescom.2005.08.010.

[31] M. P. Lazarevic, and D. L. Debeljkovi, Finite time stability analysis of linear autonomous fractional order systems with delayed state, Asian Journal of Control, 7(4), (2005), 440-447. https://doi.org/10.1111/j.1934-6093.2005.tb00407.x.

[32] M. P. Lazarevic, A. Obradovic and V. Vasic, Robust finite time stability analysis of fractional order time delay systems: New results, Advances in dynamical systems and control (2010), 101-106, retrieved from www.wsea.us/elibrary/conference/2010/Tunisia/control.

[33] T. N. Lee, and S. Diant, Stability of time delay system, IEEE Trans. Automat. Control AC 31(3), (1981), 951-953. https://doi.org/10.1109/TAC.1981.1102755.

[34] C. Li, K. Chen, J. Lu, and R. Tang, Stability and Stabilization analysis of fractional order linear systems subject to actuator saturation and distribution, IFAC paper online 50-1, (2017), 9718-9723

[35] Y. Li, Y. Chen, and I. Podlubny, Stability of fractional order nonlinear dynamic systems: Lyapunov direct method and generalized Mittag- Leffler stability, Comput. Math. Appl. 59(5), (2010), 18101821. https://doi.org/10.1016/j.camwa.2009.08.019.

[36] P. L. Liu, Exponential stability for linear time delay systems with delay dependence. Journal of the Franklin Institute, 340, (2003), 481- 488. https://doi.org/10.1016/j.jfranklin.2003.10.003.

[37] P. L. Liu, Robust exponential stabilization for uncertain systems with state and control Delay. International Journal of Systems Science, $\quad 34(12-13), \quad$ (2003), 675-682. https://doi.org/10.1080/00207720310001640719.

[38] D. Matignon, Stability results for fractional differential equations with applications to control processing. Computational Engineering in Systems Applications, (1996), 963-968

[39] F. A. Mohd, S. Manoj, and J. Renu, An application of fractional calculus in electrical engineering, Advanced Engineering Technology and Application, 5(2), (2016), 41-45. https://doi.org/10.18576/aeta/050204.

[40] S. Momani, and S. Hadid, Lyapunov stability solution of fractional integrodifferential equations, Int. J. math. Math. Sc. 47, (2004), 2503- 2507.

[41] T. Mori, Criteria for Asymptotic stability of linear time delay systems, IEEE trans. Automat. Control, AC 30, (1985), 158-161.

[42] E. Moulay, M. Dambrine, N. Yeganefar, and W. Perruquetti, Finite Time Stability and Stabilization of time delay systems. Systems and control letters, $\quad 57, \quad$ (2008), 561-566. https://doi.org/10.1016/j.sysconle.2007.12.002.

[43] M. Naber, Time fractional Schrodinger equation, J. Maths. Phys. 45, (2004), 3339-3352. https://doi.org/10.1063/1.1769611.

[44] K. B. Oldham, and J. Spanier, The fractional calculus, Academic Press, New York, (1974)

[45] I. Podlubny, Fractional differential equation, Academic Press, San Diego, (1999).

[46] Y. Ryabov, and A. Puzenko, Damped oscillation in view of the fractional oscillator equation, Phys. Rev., 66, (2002), 184-201. https://doi.org/10.1103/PhysRevB.66.184201.

[47] J. Sabatier, M. Moze, and C. Farges, Stability conditions for fractional order systems, Comput. Math. Appl. 59(5), (2010), 15941609. https://doi.org/10.1016/j.camwa.2009.08.003.

[48] S. G. Samko, A. A. Kilbas, and O. I. Marichev, Fractional integrals and derivatives theory and applications, Gordon and Breach, New York, (1993).

[49] Y. Shen, L. Zhu, and Q. Guo, Finite time boundedness analysis of uncertain neural networks with time delay: An LMI approach. Proc. 4th Int Symp. Neural Networks, Nanjing, China. (2007), 904-909. https://doi.org/10.1007/978-3-540-72383-7_105.

[50] E. Soczkiewicz, Application of fractional calculus in the theory of viscoelasticity, Molecular and Quantum Acoustics, Vol. 23, (2002), 397-404.

[51] C. Tunç, and E. Biçer, Stability to a Kind of Functional differential equations of second order with multiple delays by fixed points, Abstract and Applied Analysis, 2014(3).

[52] H. Ye, J. Gao, and Y. Ding, A generalized Gronwall inequality and its application to a fractional differential equation, J. Math. Anal. Appl., 328(2007), 1075-1081. https://doi.org/10.1016/j.jmaa.2006.05.061.

[53] F. Yu, Integrable coupling system of fractional differential equations, Eur. Phys. J. Spec. Top., (2011), 193, 27-47.

[54] M. Zavarei, and M. Jamshidi, Time Delay systems: Analysis, Optimization and Applications, North-Holland, Amsterdam, (1987).

[55] X. Zhang, Some results of linear fractional order time delay system, Appl. Math. Comp., 197, (2008), 407-411. https://doi.org/10.1016/j.amc.2007.07.069.

[56] S. Zhou, and J. Lam, Robust stabilization of delay singular systems with linear fractional parametric uncertainties. Circuits Systems $\begin{array}{llll}\text { Signal Processing, 22(6), (2003), 578-588. } & \end{array}$ https://doi.org/10.1007/s00034-003-1218-x. 